\title{
The Impacts of Platinum Diffusion to the Reverse Recovery Lifetime of a High Power Diode Devices
}

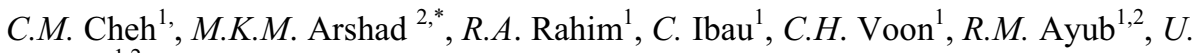 \\ Hashim $^{1,2}$ \\ ${ }^{1}$ Institute of Nano Electronic Engineering, Universiti Malaysia Perlis (UniMAP), Perlis, Malaysia \\ ${ }^{2}$ School of Microelectronic Engineering, Universiti Malaysia Perlis (UniMAP), Perlis, Malaysia
}

\begin{abstract}
The reverse recovery lifetime of a diode is one the key parameter in power electronics market. To make a diode with fast switching speed, diodes are often doped with impurities such as gold and platinum to improve its lifetime. In this works, we present the reverse recovery lifetime improvement of a power rectifier diode through platinum diffusion in the intrinsic region in between P-N junction using Design of Experiment (DOE) approach. A commercial available power rectifier is used in this study. We factored in the temperature and thermal diffusion time during the platinum diffusion process in our DOE. From results, DOE 2 (with shorter thermal duration and high temperature for diffusion) is selected based on meeting requirement for forward voltage and reverse recovery specifications i.e. forward voltage at $1.8 \mathrm{~V}$ and reverse recovery time at $27 \mathrm{~ns}$.
\end{abstract}

\section{Introduction}

The evolution of semiconductor has made power diode with high switching speeds are widely used in variety of applications such as switching the power suppliers, circuit protection, etc. There are quite a number of studies for the past decades to explain the behaviour of P-N junction diodes and also power P-I-N diode [1-14]. In diode characteristics, one of the key parameter to determine the performance of the diode is, its switching speed capability. Switching speed is defines as the time for a diode to recovers from an off-state to on-state.

Fig. 1 shows the example of reverse recovery lifetime (TRR) of a diode. In another way, TRR is also refer as the time it takes for the diode to switch from forward bias to reverse bias. The reverse recovery time is also the combination of the storage time and recovery time. During forward bias, there is a build-up of minority carriers at the edge of the depletion region. The minority will diffuse to the opposite material during reverse bias and

\footnotetext{
* Corresponding author: mohd.khairuddin@unimap.edu.my
} 
triggering the storage time. Once the minority carriers migrate they will diffuse and recombine which it is known as the recovery time.

In the past, there are various studies to discuss the reverse recovery of the diode [15-19]. Impurities such a gold and platinum can be diffused into the device body or the device body can be irradiated [20]. Irradiation is preferable due to inexpensive and is a cleaner technology. Irradiation is done at high level of energy around $1.5 \mathrm{MeV}$ to $12 \mathrm{MeV}$ to alter the device structure [21-23]...Those methods are introduced to create recombination centre that stimulate the carrier recombination and depleting the semiconductor device of mobile minority more rapidly, making the device capable to switch from off state to on state quickly. One of common trade off to have fast switching diode is the increase of leakage current and also the increase in the on state of voltage. So, to find a balance in between the main electrical parameter is mandatory when designing and fabricating the power diode. Several papers discusses about the combination of few methods to control the lifetime of a diode as narrated elsewhere [24,25].

Both platinum and gold materials are used as a reverse recovery lifetime modifiers but platinum diffusion is more commonly used due to the following factors. 1) Gold as a lifetime modifier will normally exhibit higher leakage currents compared than platinum doped diode, especially at elevated temperatures. 2) Platinum cost is lower than that of gold price. In this paper, experimental studies are conducted to find the optimum process conditions for the platinum doped method through the DOE approach on the impact of thermal duration and temperature of diffusion process. The evaluation methods are discussed in methodology section. The results of the evaluation with the discussion are further elaborate in results and discussion section.

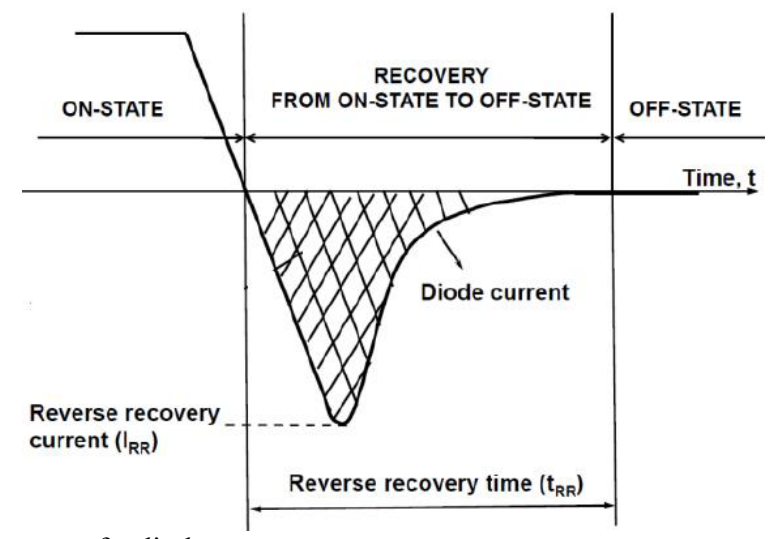

Fig. 1. The TRR diagram of a diode.

\section{Methodology}

In this research, the main interest of study is to evaluate the influences of platinum diffusion to the reverse recovery of a power rectifier. The evaluations are basically summarized in the Table 1, where eight Design Of Experiment (8 DOE) methods are applied in exploring the relationship between input $(X)$ and response $(Y)$. The input response of platinum diffusion which includes temperature of the platinum diffusion and the duration of the heat process. The output response is reverse recovery time of a power rectifier. 
Table 1. Platinum diffusion DOE Matrix.

\begin{tabular}{|c|c|c|}
\hline DOE & Thermal duration,[min.] & $\begin{array}{c}\text { Temperature, } \\
{\left[{ }^{\circ} \mathbf{C}\right]}\end{array}$ \\
\hline 1 & 0 & -1 \\
\hline 2 & -1 & 1 \\
\hline 3 & 0 & 1 \\
\hline 4 & 0 & -1 \\
\hline 5 & 0 & 1 \\
\hline 6 & 1 & -1 \\
\hline 7 & 1 & 1 \\
\hline 8 & -1 & -1 \\
\hline
\end{tabular}

The evaluation begins with fabrication of power diodes in the fab for platinum diffusion process. For thermal durations, it is represented by 0 (the standard duration), -1 (shorter duration) and 1 (longer duration). Meanwhile for temperature matrix, 1 represent high temperature and -1 represent low temperature. The fabricated devices on the wafers are then tested electrically to determine the TRR value. The reverse recovery time of the power rectifier is then analysed in the analytical software to study the relationship between input responses $(\mathrm{X})$ and output responses $(\mathrm{Y})$. Take note that the platinum diffusion process need to be carried out at the middle of fabrication since the temperature diffusion process is exceeding $800{ }^{\circ} \mathrm{C}$. Such high temperature during the diffusion process might melt the other metals of the power rectifier.

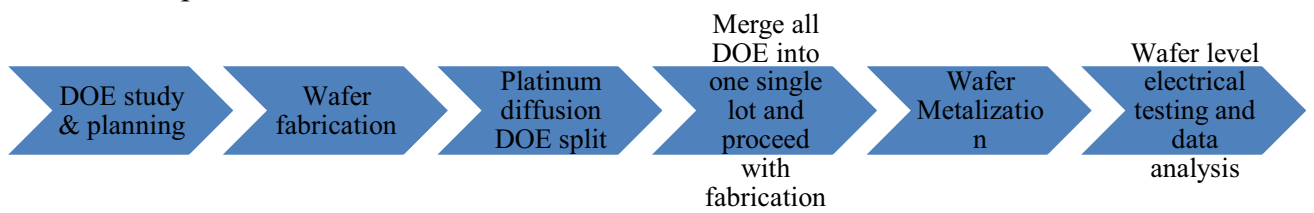

Fig. 2. The overall process flow of the evaluation.

\section{Results and Discussion}

The main objective of our work is to find a trade-off relationship between forward voltage and reverse recovery. Thus, both reverse recovery and forward voltage parameters have to take into consideration when designing a low forward voltage and fast reverse recovery time of a power rectifier. The forward voltage and reverse recovery of power rectifier is inversely proportional. When designing a fast reverse recovery time of a power rectifier, one should also consider the impact of lifetime to a forward voltage of the power rectifier. After completion of the eight DOE evaluations and the fabrication process, the wafers are then proceeds with electrical testing to obtain the electrical result.

Fig. 3 demonstrates the relationship between reverse recovery time and forward voltage of a power rectifier. The unit for forward voltage is in voltage $(\mathrm{V})$ and the reverse recovery time is in nanosecond (ns). The graph shows a negative slope, representing that the higher the forward voltage, the faster the recovery time of a power rectifier will be. Thus, it is essential to find a balance between forward voltage and reverse recovery lifetime when designing a good power rectifier. 


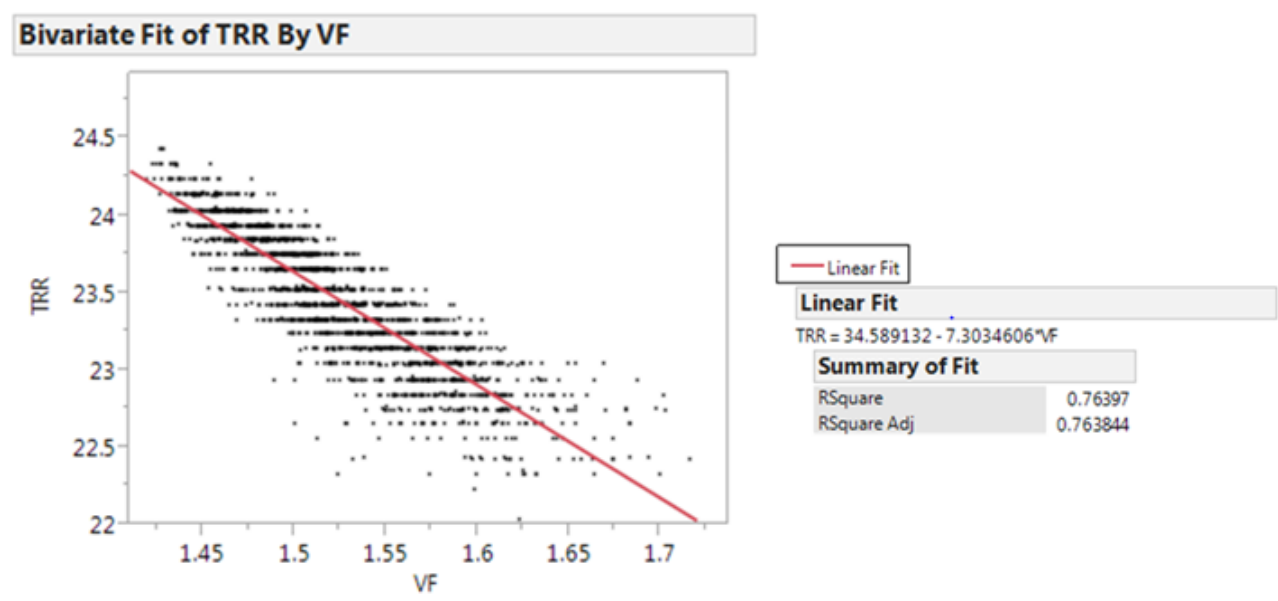

Fig.3. Relation plot between reverse recovery time (TRR) to forward voltage (VF) of a power rectifier.

Fig.4a shows the forward voltage (VF) plot for each of the DOE experiments. In each of the DOE, there are three wafers in total, which is the ideal wafer quantity to check for the variation between wafer to wafer that undergo the same process and condition. For this particular device, the forward voltage upper spec is $1.8 \mathrm{~V}$. In general, DOE number 1, 4, 6 and 8 give a superior performance of low forward voltage. Low forward voltage means lower power dissipation when the power rectifier is turn on. While in DOE number 3, 5 and 7 have higher VF distribution compared than that other DOE, with some of the points have exceed the upper spec limit of the VF. Higher VF is not desirable in the power rectifier application as it will dissipate more power during rectifier turn on. DOE 2 have a forward voltage distribution that is below the upper spec limit and have a minimum variation between wafers to wafer. In summary, if forward voltage is the only parameter that concerns, DOE number 1, 2, 4, 6 and 8 are fit for the change.

Fig. $4 \mathrm{~b}$ shows the reverse recovery time of all the 8 DOE experiments. Similar wafer splits and quantities were used as previously explained. For this device, the upper specification for reverse recovery time is 27 nanosecond. One can see that, DOE number 1 , 4,6 and 8 considered fails the specification where some of the readings exceed $27 \mathrm{~ns}$. DOE number 4, 6, 7 and 8 have most significant variation between wafer to wafer. This is not desirable as the variation is unpredictable. The wide variation may cause additional yield loss reflecting the process is unstable. On the other hand, DOE number 2, 3, 5 and 7 meet the specification where none of the readings exceed the upper spec limit. With respect to that, DOE 2 and DOE 5 provide small variation wafer to wader and fulfill TRR reading specification. 

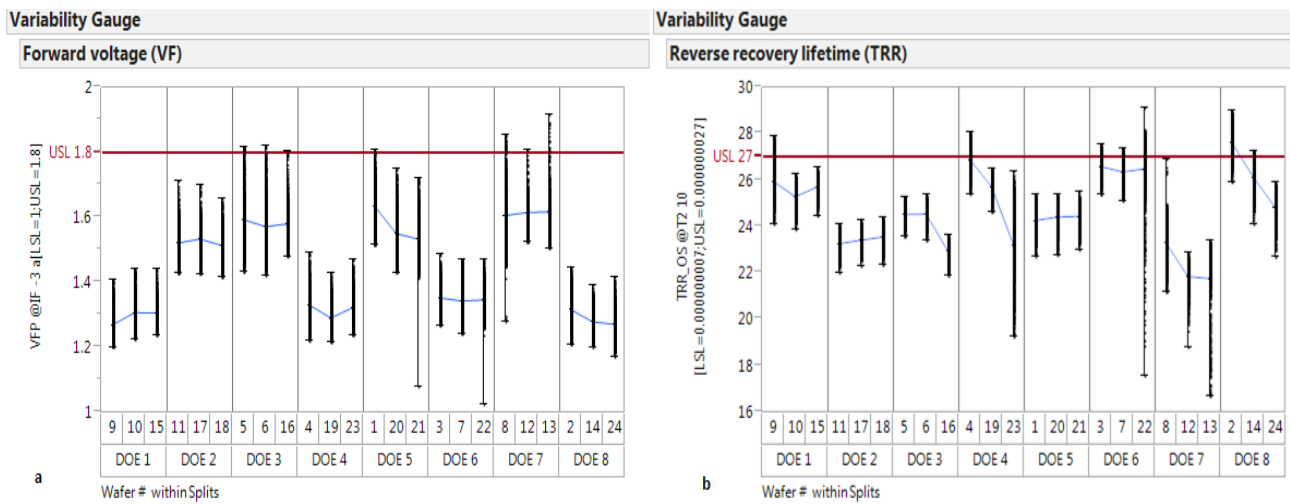

Fig. 4. a) the forward voltage (VF), b) the reverse recovery lifetime (TRR) of all 8 DOE evaluations.

The summary for each of the DOE have been tabulated in the Table 2. In conclusion, only DOE number 2 matches the requirement of forward voltage and reverse recovery time of the power rectifier. The variation between wafer to wafer in a same run is minimized compared to other splits. Although the forward voltage of the DOE number 2 is slightly higher than DOE number 1, 4, 6 and 8, but the reverse recovery time of the DOE number 2 is the best among the rest.

DOE number 1 although processed a good VF, but the reverse recovery time of a diode is not meeting the spec. The same observation goes to DOE number 4, DOE 6 and DOE 8. Despite of DOE 3 and DOE 5 gives a fast reverse recovery lifetime, but they both fail to meet the forward voltage spec at $1.8 \mathrm{~V}$. DOE number 7 is the worst, where it fails both the reverse recovery lifetime and forward voltage. In conclusion, DOE number 2 is provides the most optimum condition to produce a fast recovery lifetime and also meeting the forward voltage spec.

Fast recovery lifetime will give a high forward voltage as drawback. In this particular power rectifier, it has both tight tolerance to forward voltage and reverse recovery lifetime. The main purpose of this DOE is to explore a robust condition for platinum diffusion in order to process a good power rectifier that meets both VF and TRR requirements. From the distribution of both Fig. $4 \mathrm{a}$ and Fig. 4b, we can conclude the distribution pattern justified the hypothesis in Fig. 3, where the higher the forward voltage, the faster the recovery time of a power rectifier.

Fig. 5 shows the uniformity of the selected DOE 2 for its forward voltage and reverse recovery time. From Fig. 6, we can see there is a circular shape trend within a wafer. For forward voltage (VF), wafer edge have higher VF trend and the VF drop as it go to the centres of the wafer. The same trend observe for reverse recovery time (TRR) too where we can see a gradual increase in TRR from wafer centre to wafer edge. The uniformity for both forward voltage and reverse recovery time is acceptable where the variation within a wafer is less than $3 \mathrm{~ns}$ for reverse recovery time and $0.2 \mathrm{~V}$ for forward voltage. 
Table 2. DOE matrix for platinum diffusion study and summary of the results.

\begin{tabular}{|c|c|c|c|c|c|}
\hline DOE & $\begin{array}{c}\text { Thermal } \\
\text { duration, } \\
{[\text { min.] }}\end{array}$ & $\begin{array}{c}\text { Temperature, } \\
{\left[{ }^{\circ} \mathbf{C}\right]}\end{array}$ & $\begin{array}{c}\text { Forward } \\
\text { voltage, } \\
\text { VF }\end{array}$ & Reverse recovery lifetime, TRR & Conclusion \\
\hline 1 & 0 & -1 & $\sqrt{ }$ & $\mathrm{x}$ & Fail \\
\hline 2 & -1 & 1 & $\sqrt{ }$ & $\mathrm{V}$ & Selected \\
\hline 3 & 0 & 1 & $\mathrm{x}$ & $\mathrm{x}$ & Fail \\
\hline 4 & 0 & -1 & $\sqrt{ }$ & $\sqrt{ }$ & Fail \\
\hline 5 & 0 & 1 & $\mathrm{x}$ & $\mathrm{x}$ & Fail \\
\hline 6 & 1 & -1 & $\mathrm{x}$ & $\mathrm{x}$ & Fail \\
\hline 7 & 1 & 1 & $\sqrt{ }$ & $\mathrm{x}$ & Fail \\
\hline 8 & -1 & -1 & & & \\
\hline
\end{tabular}
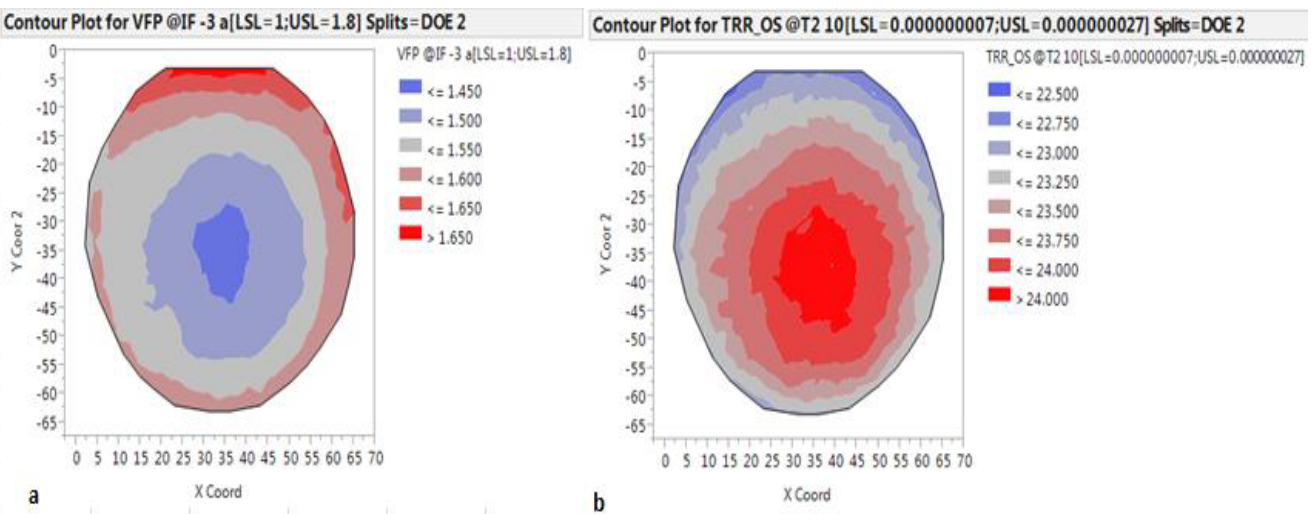

Fig.5. The contour plot of DOE number 2 to check for uniformity within a wafer for a) forward voltage (VF) and b) reverse recovery time (TRR).

\section{Conclusions}

Through the understanding of reverse recovery lifetime of a diode, we have further explore the details working principle of platinum diffusion towards to the influence of reverse recovery lifetime of a diode. Platinum diffusion is the optimum method to control the TRR of diode compared with gold diffusion due to it is more economical and lower leakage current drop. In this paper, we have explained the details of platinum diffusion factors that influence the output response of reverse recovery lifetime of a diode. DOE number 2 provides the optimum matrix to process good forward voltage and fast reverse recovery time for a power rectifier where it meets the given specification for VF below $1.8 \mathrm{~V}$ and TRR below $27 \mathrm{~ns}$.

\section{References}

1. S. Sze, Physics of Semiconductor Devices, (Wiley, New York 1969)

2. R. Kingston, Proc. IRE, 28, 829 (1954)

3. J.L. Moll, Physics of Semiconductor, (McGraw-Hill, New York 1964)

4. R.N. Hall, Power Rectifiers and Transistors, 80, 1512 (1952)

5. S.K. Ghandhi, Semiconductor of Power device, (Wiley, New York 1977)

6. S. Member, IEEE Trans. on Electron Devices, 43, 142 (1996) 
7. B. Mazhari, M. Sinha, J. Dixit, IEEE Conference on Electron Devices and Solid-State Circuits (2005)

8. M. Tsukuda, Y. Sakiyama, H. Ninomiya, M. Yamaguchi, $21^{\text {st }}$ International Symposium on Power Semiconductor Devices \& IC's (2009)

9. B.J. Baliga, Modern Power Devices, (John Wiley \& Sons Inc., 1987)

10. W.M. Jubadi, S.N.M. Noor, IEEE Symp. Ind. Electron. Appl., 428 (2010)

11. E. Abiri, M.R. Salehi, S. Kohan, M. Mirzazadeh, IEEE Second Pacific-Asia Conf. Circuits, Commun. Syst., 60 (2010)

12. W. Jablonski, 12th International Conference on Microwaves and Radar, MIKON'98, 69 (1998)

13. S. Sawant, B. Baliga, Power Semiconductor Devices and ICs, 1999. ISPSD'99. Proceedings, IEEE The 11th International Symposium, 153 (1999)

14. N.I. Shuhaimi, M. Mohamad, W.M. Jubadi, R.Tugiman, N. Zinal, R.M. Zin, IEEE International Conference on Semiconductor Electronics, 12 (2010)

15. E. Napoli, A.G.M Strollo, P. Spirito., IEEE Transactions on Power Electronics 14, 25 (1997)

16. A.G .Rojas-Hernández, A. Vera-Marquina, A. Garcia-Juárez, IEEE Electronics, Robotics and Automotive Mechanics Conference (CERMA), 389 (2012)

17. V.A Temple, F.W. Holroyd, IEEE Transactions on electron Devices, 30(7), 782 (1983)

18. J. Vobecky, S. Member, V. Zahlava, P. Hazdra, IEEE Electron Device Letters, 35(3), 375 (2014)

19. B.J. Baliga, E. Sun , IEEE Trans. Electron Devices, 24, 685 (1977)

20. E. Iliescu, V. Banu, A. Niculescu, 12th International Conference on High-Power Particle Beams, 2, 2 (1998)

21. R.O. Carlson, Y.S. Sun, H.B. Assalit, IEEE Trans. Electron Devices, 24, 8 (1977)

22. W. Itthikusumarn, W. Jakphet, W. Titiroongruang, International Symposium on NextGeneration Electronics (ISNE), 7 (2015)

23. D. Hu, J.K.O. Sin, B. Kang, X. Cheng, Y. Wu, S. Xie, IEEE Southern Power Electronics Conference SPEC. (2007)

24. J. Vobecky, P. Hazdra, European Conference on Power Electronics and Applications, (2005) 